Article

\title{
Organic Hollow Mesoporous Silica as a Promising Sandalwood Essential Oil Carrier
}

\author{
Zuobing Xiao, Heqing Bao, Shuhan Jia, Yutian Bao, Yunwei Niu and Xingran Kou * \\ School of Perfume and Aroma Technology, Shanghai Institute of Technology, Shanghai 201418, China; \\ xzb@sit.edu.cn (Z.X.); 186071202@mail.sit.edu.cn (H.B.); 186071207@mail.sit.edu.cn (S.J.); \\ 186071201@mail.sit.edu.cn (Y.B.); nyw@sit.edu.cn (Y.N.) \\ * Correspondence: kouxr@sit.edu.cn; Tel.: +86-021-60877237
}

check for updates

Citation: Xiao, Z.; Bao, H.; Jia, S.; Bao, Y.; Niu, Y.; Kou, X. Organic Hollow Mesoporous Silica as a Promising Sandalwood Essential Oil Carrier. Molecules 2021, 26, 2744. https://doi.org/10.3390/ molecules26092744

Academic Editor: Farid Chemat

Received: 10 March 2021

Accepted: 3 May 2021

Published: 7 May 2021

Publisher's Note: MDPI stays neutral with regard to jurisdictional claims in published maps and institutional affiliations.

Copyright: (C) 2021 by the authors. Licensee MDPI, Basel, Switzerland. This article is an open access article distributed under the terms and conditions of the Creative Commons Attribution (CC BY) license (https:// creativecommons.org/licenses/by/ $4.0 /)$.

\begin{abstract}
As film-forming agents, fillers and adsorbents, microplastics are often added to daily personal care products. Because of their chemical stability, they remain in the environment for thousands of years, endangering the safety of the environment and human health. Therefore, it is urgent to find an environmentally friendly substitute for microplastics. Using $n$-octyltrimethoxysilane (OTMS) and tetraethoxysilane (TEOS) as silicon sources, a novel, environmentally friendly, organic hollow mesoporous silica system is designed with a high loading capacity and excellent adsorption characteristics in this work. In our methodology, sandalwood essential oil (SEO) was successfully loaded into the nanoparticle cavities, and was involved in the formation of Pickering emulsion as well, with a content of up to $40 \%(w / w)$. The developed system was a stable carrier for the dispersion of SEO in water. This system can not only overcome the shortcomings of poor water solubility and volatility of sandalwood essential oil, but also act as a microplastic substitute with broad prospects in the cosmetics and personal care industry, laying a foundation for the preparation and applications of high loading capacity microcapsules in aqueous media.
\end{abstract}

Keywords: sandalwood essential oil; organic hollow mesoporous silica; Pickering emulsion; loading capacity

\section{Introduction}

In recent years, natural green additives used in daily chemical products have attracted immense interest. Natural plant essential oils, known as liquid gold, are a type of plantderived secondary metabolites with small molecular weight steamed with water vapour. They have biodegradability and excellent antioxidant, antibacterial and other biological activities [1,2]. Therefore, they can find interesting applications in medicine, food and the daily chemical industry. Sandalwood (Santalum album L.), found in India, Malaysia, Australia and Indonesia, is considered to be one of the most valuable trees in the world. Sandalwood essential oil (SEO), obtained from the roots and trunk of sandalwood, is an industrial product widely used in medicine, chemicals and spices. SEO not only has bactericidal, antibacterial and antitumour functions, but also can relax nerves and relieve stress. Thus, it is widely used in cosmetics, perfumes and personal care products [3]. However, the application of SEO is greatly limited owing to its volatilisation, low water solubility and poor stability.

Nano-/microcapsule technology has the potential to overcome these problems. In the food industry, Eucalyptus staigeriana essential oil was loaded using cashew gum as wall material by Paula to increase its antibacterial activity [4]. The clove oil microcapsules were prepared by Wang [5]. Compared with clove oil, the microcapsules exhibited strong heat resistance and excellent antiseptic properties on meat products. Microcapsules with thyme oil as core material and maltodextrin as wall material were prepared by spray-drying [6] The results indicated that the microcapsules had antibacterial effects on Vibrio alginolyticus and Vibrio parahaemolyticus. Nano-/microcapsule technology uses carriers to encapsulate 
unstable, reactive and volatile liquids or solids to form particles within a size range of $1 \mathrm{~nm}-1 \mathrm{~mm}$. According to the type of materials, microcapsule carriers can be classified into polymer, inorganic and polymer-inorganic microcapsules [7]. Polymer microcapsules, such as polyethene and oxidised polyethene, are often added to personal care products, including toothpaste, detergents, facial cleansers and scrubs [8]. Nevertheless, due to their chemical stability, corrosion resistance and lack of biodegradation, they remain in the environment for hundreds and even thousands of years, seriously endangering the safety of the environment and life [9]. Consequently, there is an urgent need to find an environmentally friendly alternative to plastic microcapsules with a certain degree of friction resistance.

Silicon dioxide naturally exists in nature; the synthesis of mesoporous silica nanoparticles (MSNs) can be traced back to the early 1990s, and remarkable progress has been made since then $[10,11]$. Owing to their nontoxicity, high specific surface area, regular pore structure, adjustable pore size distribution, easy functionalisation, excellent mechanical stability and biocompatibility [12-15], MSNs have been widely used in spices [16], medicine [17], agriculture [18] and catalysts [19] as microencapsulated nanocarriers. On the contrary, their applications have been limited, due to their low loading capacity [20]. Hollow MSNs may be particularly valuable, due to their large internal space and pore structure, which can provide high loading capacity and slow-release characteristics for guest molecules [21-24].

Despite impressive achievements, the encapsulation of guest molecules in hollow silica materials is mainly achieved by the traditional impregnation method, which requires high hydrophilic and hydrophobic properties of encapsulated guest molecules. Due to the poor impregnation effect of hydrophobic guest materials, it is quite difficult for a large number of guest molecules to enter the cavity. Although the addition of cosolvents or phase transfer agents can enhance packaging efficiency, the introduction of supplementary additives can violate the principles of green chemistry.

Introducing hydrophobic groups on the surface of solid materials is beneficial to increase their hydrophobic properties, and thus, strengthen their adsorption to hydrophobic organic compounds in water [25-27]. Raspberry-like superhydrophobic hollow silica particles, which are modified by nonafluorohexyltriethoxysilane (NFH-silane) to reduce their surface energy, were obtained by Park and co-workers [28]. Unfortunately, although their adsorption properties can be increased, fluorine-containing modifiers are expensive and harmful to the environment [29]. Xu prepared a series of hydrophobic hollow silica nanospheres with $n$-octyltrimethoxysilane and other hydrophobic modifiers by the inverse emulsion method and selective etching [30]. However, stronger hydrophobicity of the hollow silica nanospheres was prone to result in a decreased dispersion in water, even lead to a float on water, which is a disadvantage for practical application. Moreover, stronger hydrophobicity does not always mean higher loading capacity, since the reduced dispersibility in water decreased their accessibility to the cargo compounds dissolved in water [31]. Therefore, adjusting the dispersion and distribution of hollow mesoporous silica in aqueous systems should not be neglected.

In this paper, novel hollow mesoporous silica with a high loading capacity and superior adsorption was designed using $n$-octyltrimethoxysilane (OTMS) and tetraethoxysilane (TEOS) as silicon sources. SEO was not only loaded in the nanoparticle cavities, but also revolved in Pickering emulsion as the dispersed phase. The effect of the amount of silicon source deposited on the shell, the additional amount of ammonium hydroxide solution, the proportion of OTMS to the silicon source and the additional amount of CTAC (cetyltrimethylammonium chloride) on the water contact angle, the dispersion of organic hollow mesoporous silica in water and the loading capacity of SEO were investigated, and organic hollow mesoporous silicas with high loading capacity and superior dispersion were prepared. The hollow mesoporous silica particles can substitute microplastics, making them attractive for applications in cosmetics and personal care products. 


\section{Results and Discussion}

\subsection{Experimental Preparation Process}

In this study, Scheme 1 illustrates the experimental methods. First, monodisperse silica nanospheres were synthesised by the hydrolysis of TEOS and the cross-linking of silicate. Then, the pore structure-directing agent CTAC was added to the solution. Considering that CTAC had both hydrophobic chains and hydrophilic groups, it can be arranged directionally to form micelles in the system, which can be radially adsorbed on the outer surface of negatively charged silica nanospheres by electrostatic adsorption. Consequently, the mixture of TEOS and OTMS was added, and the organic part of OTMS entered into CTAC micelles and enlarged the micelles [32]. Simultaneously, under the catalysis of the alkaline condition, the silicon source was hydrolysed and polymerised with positively charged CTAC. The change in charge density made the long chains of CTAC close to each other and further promoted the combination of the silicon source and the pore structuredirecting agent. After condensation, organic bilayer silica microspheres were formed layer-by-layer on the surface of silica nanospheres. Furthermore, the organic bilayer silica microspheres were etched by a sodium carbonate aqueous solution at $80^{\circ} \mathrm{C}$. According to the research results of the Shi group [33,34], the degree of condensation between the inner cores of pure silica was lower than that of hybrid silica shells. Meanwhile, due to the protection of the hydrophobic layer on the surface of the hybrid shell, sodium carbonate selectively etched the core to form a hollow structure. CTAC played a key role in the formation of organic hollow mesoporous silica. However, it must be removed since it had a certain degree of cytotoxicity. The main methods of removing CTAC were extraction and calcination. But calcination required high temperatures $\left(600^{\circ} \mathrm{C}\right)$ for more than $4 \mathrm{~h}$, causing the hollow structure to collapse. Therefore, reflux extraction with acid and alcohol was performed to remove CTAC and obtain organic hollow mesoporous silica connected by nanopores. SEO was added after the organic hollow mesoporous silica was uniformly dispersed in water. The adsorption capacity of SEO was enhanced, due to the hydrophobicity of the hybrid organic shell. After continuous stirring, SEO was adsorbed on the surface of the internal cavity of organic hollow mesoporous silica. This system formed a solution of oil in water, known as Pickering emulsion. Therefore, the loading capacity of organic hollow mesoporous silica greatly enhanced.

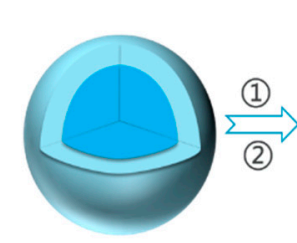

organic bilayer silica microspheres

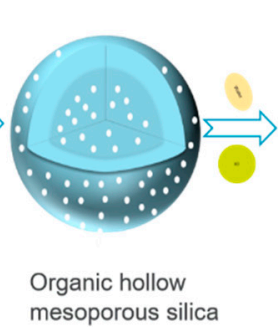

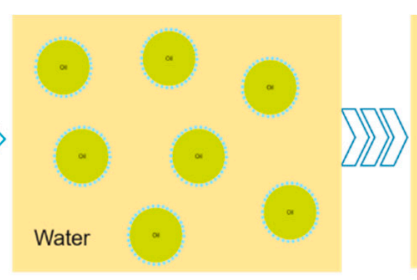

Pickering emulsion

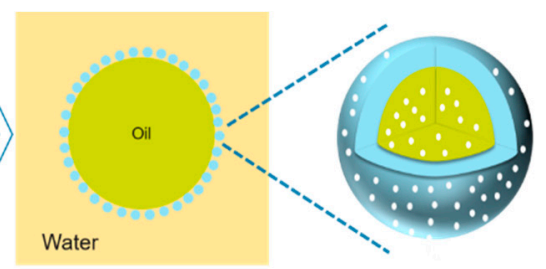

Oil/ water emulsion
Oil-loaded organic hollow mesoporous silica

Scheme 1. The preparation process of the whole experiment. (1) etched by sodium carbonate aqueous solution. (2) removing CTAC with acid and alcohol solution.

2.2. The Effects of Different Factors on the Water Contact Angle of Organic Hollow Mesoporous Silica and Its Loading Capacity to SEO

The loading capacity of Pickering emulsion to SEO was used as an indicator for the single factor test. According to the results of the pre-experiment, the $3 \mathrm{~mL}$ of ammonium hydroxide solution, 30\% (v/v) of OTMS to silicon source deposited on the shell and $335 \mathrm{mg}$ of CTAC were selected for the following tests, respectively.

2.2.1. Effect of the Amount of Silicon Source Deposited on the Shell on the Water Contact Angle of Organic Hollow Mesoporous Silica and Its Loading Capacity to SEO

First of all, the effect of the amount of silicon source deposited on the shell on the water contact angle (WCA) of organic hollow mesoporous silica and its loading capacity to SEO was investigated. The addition amounts of silicon source deposited on the shell were 
$1.5 \mathrm{~mL}, 2.5 \mathrm{~mL}, 3.5 \mathrm{~mL}, 4.5 \mathrm{~mL}$ and $5.5 \mathrm{~mL}$, respectively. The significant difference in WCA in Figure 1a reflected the difference in the wettability of solid materials obtained. With the increase in the amount of silicon source deposited on the shell, the WCA increased. The change of WCA may be caused by octyl groups [31]. In order to confirm our conjecture, FTIR was used to characterise the organic hollow mesoporous silica formed by the silicon source deposited on the shell with different addition amounts (Figure 2a). The absorption peaks around $2928.6 \mathrm{~cm}^{-1}$ and $2858.2 \mathrm{~cm}^{-1}$ were attributed to the antisymmetric and symmetrical stretching vibration of the functional group $-\mathrm{CH}_{2}$. The changes at $1464.5 \mathrm{~cm}^{-1}$ may be attributed to the asymmetric angular vibration of $\mathrm{CH}_{3} \cdot-\mathrm{CH}_{2}-$ and $\mathrm{CH}_{3}$ were characteristic functional groups of OTMS. Their vibration amplitude gradually increased with the increase of the amount of silicon source deposited on the shell. When the mixed silicon source was added to the system, due to the hydrophobic silicon source possessing a nonpolar head, it was preferentially incorporated into the interior of CTAC. At this time, a small amount of TEOS was not involved in the reaction. With the increase in silicon sources, more octyl silicon sources were hybridised into the shells, and less TEOS entered into the shells. Therefore, the WCA increased with the increase of the amount of silicon source deposited on the shell.
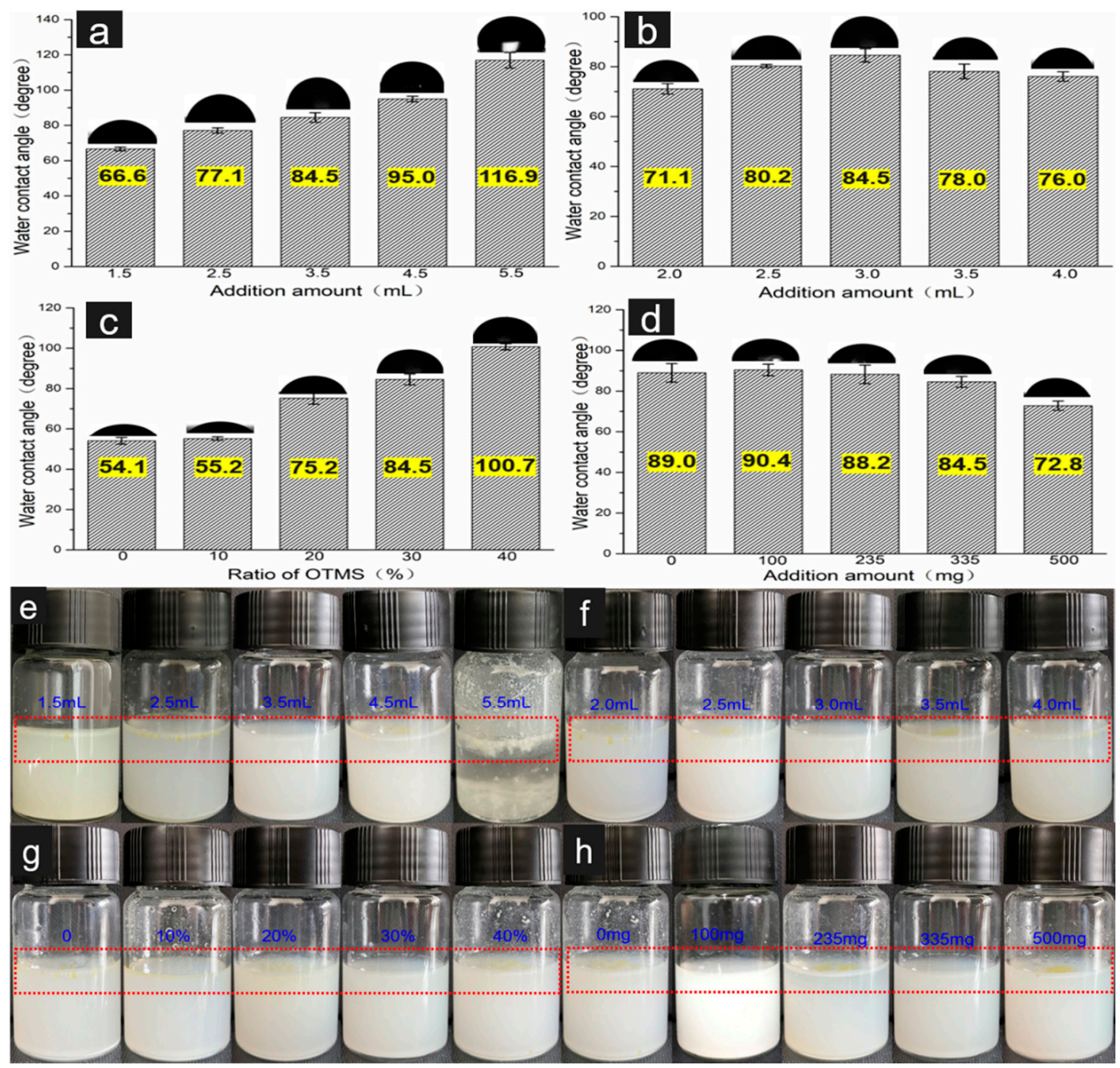

Figure 1. The effects of different factors on the water contact angle of organic hollow mesoporous silica and its loading capacity to SEO (a,e) The additional amount of silicon source deposited on the shell were $1.5 \mathrm{~mL}, 2.5 \mathrm{~mL}, 3.5 \mathrm{~mL}, 4.5 \mathrm{~mL}$ and $5.5 \mathrm{~mL}$ from left to right. $(\mathbf{b}, \mathbf{f})$ The ammonia hydroxide solution addition were $2.0 \mathrm{~mL}, 2.5 \mathrm{~mL}, 3 \mathrm{~mL}, 3.5 \mathrm{~mL}$ and $4 \mathrm{~mL}$ from left to right. (c,g) The ratio of OTMS to silicon sources deposited on the shell were $0,10 \%, 20 \%, 30 \%$ and $40 \%(v / v)$ from left to right. (d,h) The additional amount of CTAC were $0,100 \mathrm{mg}, 235 \mathrm{mg}, 335 \mathrm{mg}$ and $500 \mathrm{mg}$ from left to right. 

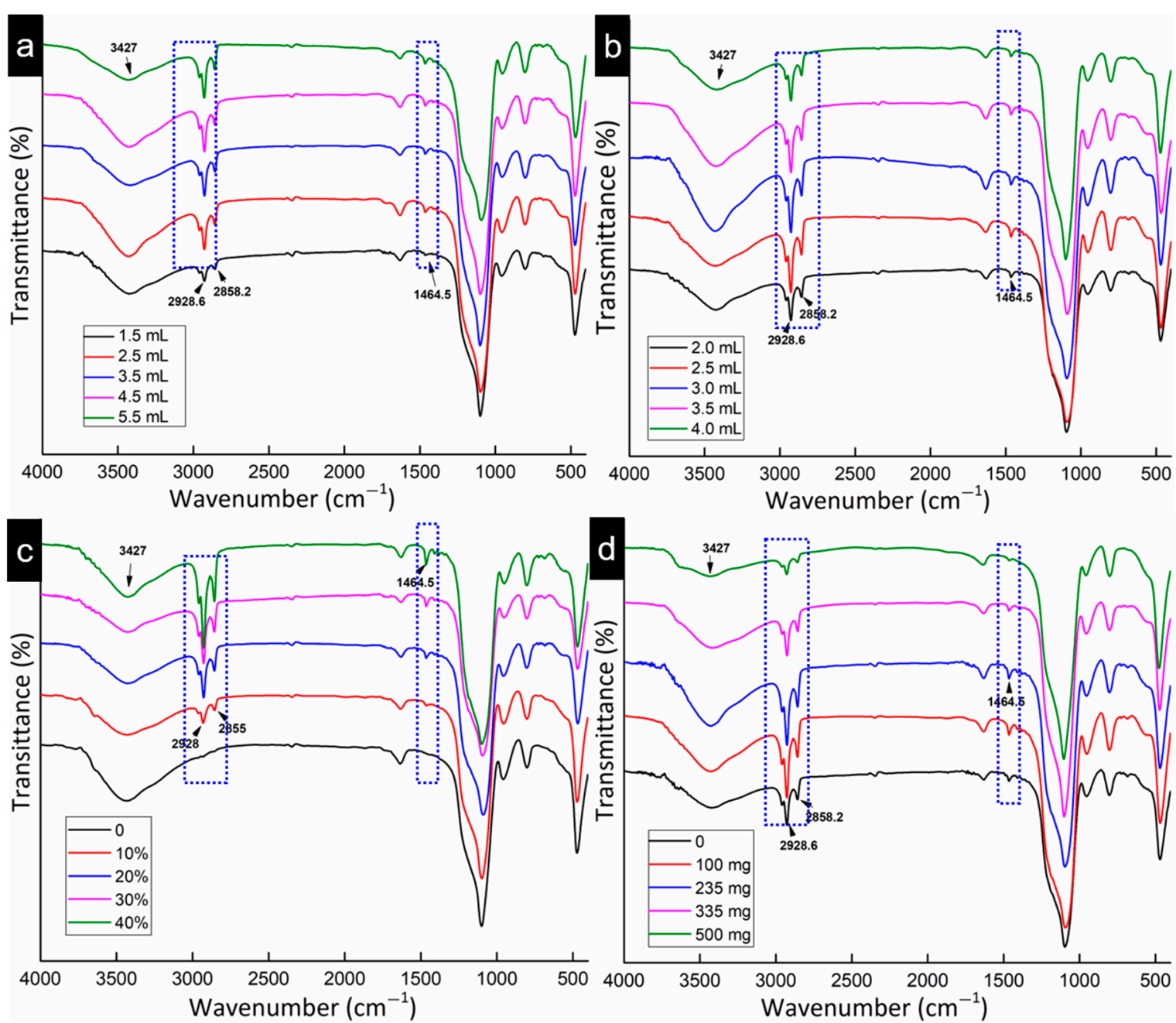

Figure 2. FTIR of organic hollow mesoporous silica formed by different influencing factors (a) The additional amount of silicon source deposited on the shell were $1.5 \mathrm{~mL}, 2.5 \mathrm{~mL}, 3.5 \mathrm{~mL}, 4.5 \mathrm{~mL}$ and $5.5 \mathrm{~mL}$. (b) The ammonia hydroxide solution addition were $2.0 \mathrm{~mL}, 2.5 \mathrm{~mL}, 3 \mathrm{~mL}, 3.5 \mathrm{~mL}$ and $4 \mathrm{~mL}$. (c) The ratio of OTMS to silicon sources deposited on the shell were 0 , $10 \%, 20 \%, 30 \%$ and $40 \%$. (d) The additional amount of CTAC were $0,100 \mathrm{mg}, 235 \mathrm{mg}, 335 \mathrm{mg}$ and $500 \mathrm{mg}$.

Then $0.03 \mathrm{~g}$ of SEO was added to the aqueous solution of organic hollow mesoporous silica. The results showed that when the amount of silicon source deposited on the shell was $3.5 \mathrm{~mL}$, the dispersion of the system was good, and no free-oil was observed (Figure 1e). This indicated that all the essential oils were embedded in organic hollow mesoporous silica. The SEO loading capacity was the highest when the addition of the silicon source deposited on the shell was $3.5 \mathrm{~mL}$. The amount of SEO might be affected by the thickness of the organic hollow mesoporous silica shell. When the addition of silicon source was less than $3.5 \mathrm{~mL}$, the spherical shell formed was so thin that it could not form a complete sphere, thus causing the structure to collapse. At the same time, due to its poor adsorption performance, the SEO could not actively enter the cavity, resulting in low loading capacity. Thus, it formed the free-oil. When the amount of silicon source deposited on the shell was greater than $3.5 \mathrm{~mL}$, the WCA was greater than $90^{\circ}$ (Figure 1a). However, because the spherical shell was too thick to enter the cavity, SEO was trapped in the mesoporous channel of the spherical shell. It was difficult to load a large amount of SEO. Therefore, the additional amount of silicon source deposited on the shell was selected as $3.5 \mathrm{~mL}$ to carry out the follow-up experiment. 
2.2.2. Effect of Ammonium Hydroxide Solution Addition on the WCA of Organic Hollow Mesoporous Silica and Its Loading Capacity to SEO

Secondly, the effect of ammonium hydroxide solution addition on the WCA of organic hollow mesoporous silica and its loading capacity to SEO was investigated. The addition amounts of ammonium hydroxide solution were $2 \mathrm{~mL}, 2.5 \mathrm{~mL}, 3 \mathrm{~mL}, 3.5 \mathrm{~mL}$ and $4 \mathrm{~mL}$, respectively. As a catalyst, the amount of ammonium hydroxide solution had an important effect on the degree of the hydrolysis and condensation reaction of TEOS and OTMS. It can be seen from Figure $1 \mathrm{~b}$ that the WCA of organic hollow mesoporous silica increased first and then decreased with an increase in the volume of ammonium hydroxide solution, which may be because ammonia as a catalyst had a great influence on the hydrolysis and condensation of organosilanes. It impacted the formation and accumulation rate of silane on the shell, thereby affecting the WCA. The FTIR (Figure 2b) was used to analyse the organic hollow mesoporous silica formed by different amounts of ammonium hydroxide solution. The vibration amplitude of the marked place increased first, and then decreased with an increase in the amount of ammonia addition, which was consistent with the results of the WCA, proving that the content of octyl functional groups in organic hollow mesoporous silica increased at first and then decreased. When the amount of ammonium hydroxide solution was less than $3.0 \mathrm{~mL}$, the hydrolysis condensation rate increased with the increase in the ammonia volume, so the WCA increased gradually. When the volume of ammonium hydroxide solution exceeded $3.0 \mathrm{~mL}$, the hydrolysis and condensation occurred not only at the oil-water interface, but also in the water phase, producing free silica particles and resulting in smaller WCA.

Subsequently, $0.03 \mathrm{~g}$ of SEO was added to the aqueous solution of organic hollow mesoporous silica. Figure $1 \mathrm{f}$ clearly showed that when the amount of ammonia was $3 \mathrm{~mL}$, no free-oil was observed. This meant that the loading capacity of SEO was the highest when the amount of ammonium hydroxide solution was $3.0 \mathrm{~mL}$. The most likely reason was that when the amount of ammonium hydroxide solution was $3.0 \mathrm{~mL}$, the WCA of the obtained solid material was the largest (Figure 1b). As a result of the strongest adsorption at this time, the loading capacity of SEO was the highest. Consequently, the additional amount of ammonia was selected as $3 \mathrm{ml}$ to carry out the follow-up experiment.

2.2.3. Effect of the Ratio of OTMS to the Silicon Source Deposited on the Shell on the WCA of Organic Hollow Mesoporous Silica and Its Loading Capacity to SEO

Thirdly, the effect of the ratio of OTMS to the silicon source deposited on the shell on the WCA of organic hollow mesoporous silica and its loading capacity to SEO was investigated. The proportions of OTMS to the silicon source deposited on the shell were $0,10 \%, 20 \%, 30 \%$ and $40 \%$, respectively. OTMS was a hydrophobic organosilicon source, which was hydrolysed and condensed with TEOS in the presence of ammonia to form a hybrid shell of organic hollow mesoporous silica. The ratio of OTMS to the silicon source had an important influence on the WCA of organic hollow mesoporous silica. In Figure 1c, the WCA of organic hollow mesoporous silica increased with the increase of the proportion of OTMS. The reason may be that OTMS was introduced into the surface of organic hollow mesoporous silica to form nanosised papillae, increasing the surface roughness of the material. At the same time, FTIR was used to characterise the organic hollow mesoporous silica formed by adding different OTMS ratios (Figure 2c). When OTMS was not added, no vibration was found at the $2928 \mathrm{~cm}^{-1}, 2855 \mathrm{~cm}^{-1}$ and $1464 \mathrm{~cm}^{-1}$. With the increasing of OTMS, the amplitude of the vibration of the effective functional group became larger and larger. The fact that the formed organic hollow mesoporous silica contained more and more octyl groups was effectively confirmed. Therefore, the hydrophobic effect of the particles became stronger with an increase in the proportions of silicon sources deposited on the shell occupied by OTMS.

OTMS affected the WCA of organic hollow mesoporous silica, and thus, the loading capacity of SEO. Then $0.03 \mathrm{~g}$ SEO was added to the aqueous solution of organic hollow mesoporous silica. It was found that when the proportion of OTMS to the silicon source deposited on the shell was 30\% (v/v), no free-oil was observed (Figure $1 \mathrm{~g}$ ). It may be that 
when the ratio of OTMS to silicon source deposited on the shell was less than $30 \%(v / v)$, the hydrophilicity of the formed organic hollow mesoporous silica was so high that the SEO cannot enter its internal cavities actively. When the proportion of OTMS to silicon source deposited on the shell was $40 \%(v / v)$, the WCA was $100.7^{\circ}$ (Figure 1c). At this time, the surface energy of organic hollow mesoporous silica was too large to contact part of SEO dissolved in water. The loading capacity was relatively small, so some free-oil appeared. When the ratio of OTMS to silicon source deposited on the shell was 30\% (v/v), the WCA was $85.4^{\circ}$. The surface energy and adsorption capacity were relatively good, so the loading capacity of SEO was larger. Therefore, the proportion of OTMS to the silicon source deposited on the shell was selected as $30 \%(v / v)$ to carry out the follow-up experiment.

\subsubsection{Effect of the Additional Amount of CTAC on the WCA of Organic Hollow} Mesoporous Silica and Its Loading Capacity to SEO

Fourth, the effect of the amount of CTAC on the WCA of organic hollow mesoporous silica and its loading capacity to SEO was investigated. The additions of CTAC were 0, $100 \mathrm{mg}$, $235 \mathrm{mg}$, $335 \mathrm{mg}$ and $500 \mathrm{mg}$, respectively. Figure 1d shows the variation of the WCA of organic hollow mesoporous silica with the addition of CTAC. When the addition was less than $235 \mathrm{mg}$, the WCA of the organic hollow mesoporous silica mildly changed. The reason may be that CTAC as a surfactant affected the assembly of silicon sources. Due to the low concentration of CTAC at this time, the shell of the silicon source condensed into a saturated state after the addition of the silicon source. As a result, the WCA changed small. When the amount of CTAC continued to increase, the WCA of solid materials decreased. The reason may be that CTAC not only participated in the formation of pores, but also the excess CTAC and hydrophobic OTMS assembled to form polar headoutward hydrophilic molecules, resulting in the decrease of WCA. From the FTIR spectrum (Figure $2 \mathrm{~d}$ ), it can be seen that with the increase in the amount of CTAC, the vibration amplitude of effective functional groups increased at first and then decreased. This result was consistent with the results of WCA, indicating that the content of octyl functional groups in organic hollow mesoporous silica increased at first and then decreased.

CTAC impacted the pore formation of hollow mesoporous silica [35], and further affected its loading capacity. Subsequently, $0.03 \mathrm{~g}$ SEO was added to the aqueous solution of organic hollow mesoporous silica. The results showed that when the additional amount of CTAC were $100 \mathrm{mg}$ and $335 \mathrm{mg}$, no free-oil was observed (Figure $1 \mathrm{~h}$ ). When the addition of SEO reached $0.04 \mathrm{~g}$, free-oil appeared in the sample with CTAC addition of $335 \mathrm{mg}$ (Figure 3b). Therefore, when the addition of CTAC was $100 \mathrm{mg}$, the prepared organic hollow mesoporous silica had the best loading capacity and dispersion. In order to measure the loading capacity, the addition of SEO was further increased to $0.045 \mathrm{~g}$. It was found that once $0.045 \mathrm{~g}$ of SEO was added, free-oil appeared in the sample (Figure 3c). According to the calculation formula of the loading capacity:

$$
\mathrm{LC}(\%)=\frac{\mathrm{w}_{1}}{\mathrm{w}_{1}+\mathrm{w}_{2}} \times 100 \%
$$

Among them, LC (\%) is loading capacity of organic hollow mesoporous silica to SEO, $\mathrm{w}_{1}$ is maximum addition amount of SEO when free-oil did not appear, $\mathrm{w}_{2}$ is addition amount of organic hollow mesoporous silica.

Thus, the loading capacity of organic hollow mesoporous silica to SEO was $40 \%$ (w/w). Compared with non-biodegradable materials, such as polyacrylamide and polymethyl methacrylate, the load may not be large enough, but it has a bright future and great potential [36]. Notably, the hollow mesoporous silica formed by 100-mg CTAC had the highest loading capacity of SEO, which may be because CTAC micelles affected the formation of mesoporous silica. With the increasing addition of CTAC, the mesopores became smaller, and their loading capacity decreased. When the WCA was $90.4^{\circ}$ (Figure 1d), SEO was not only adsorbed into the internal cavity of organic hollow mesoporous silica, but also formed Pickering emulsion with organic hollow mesoporous silica. In order to confirm 
our conjecture, the emulsion was observed by an Olympus microscope and confocal laser scanning microscope. As shown in Figure 3d, obvious milk droplets can be seen without adding any surfactant. Meanwhile, under the laser confocal microscope (Figure 3e,f), it was observed that SEO labelled with Nile red and organic hollow mesoporous silica formed oil in water $(\mathrm{O} / \mathrm{W})$ Pickering emulsion. Therefore, the SEO loading capacity of organic hollow mesoporous silica was the highest.

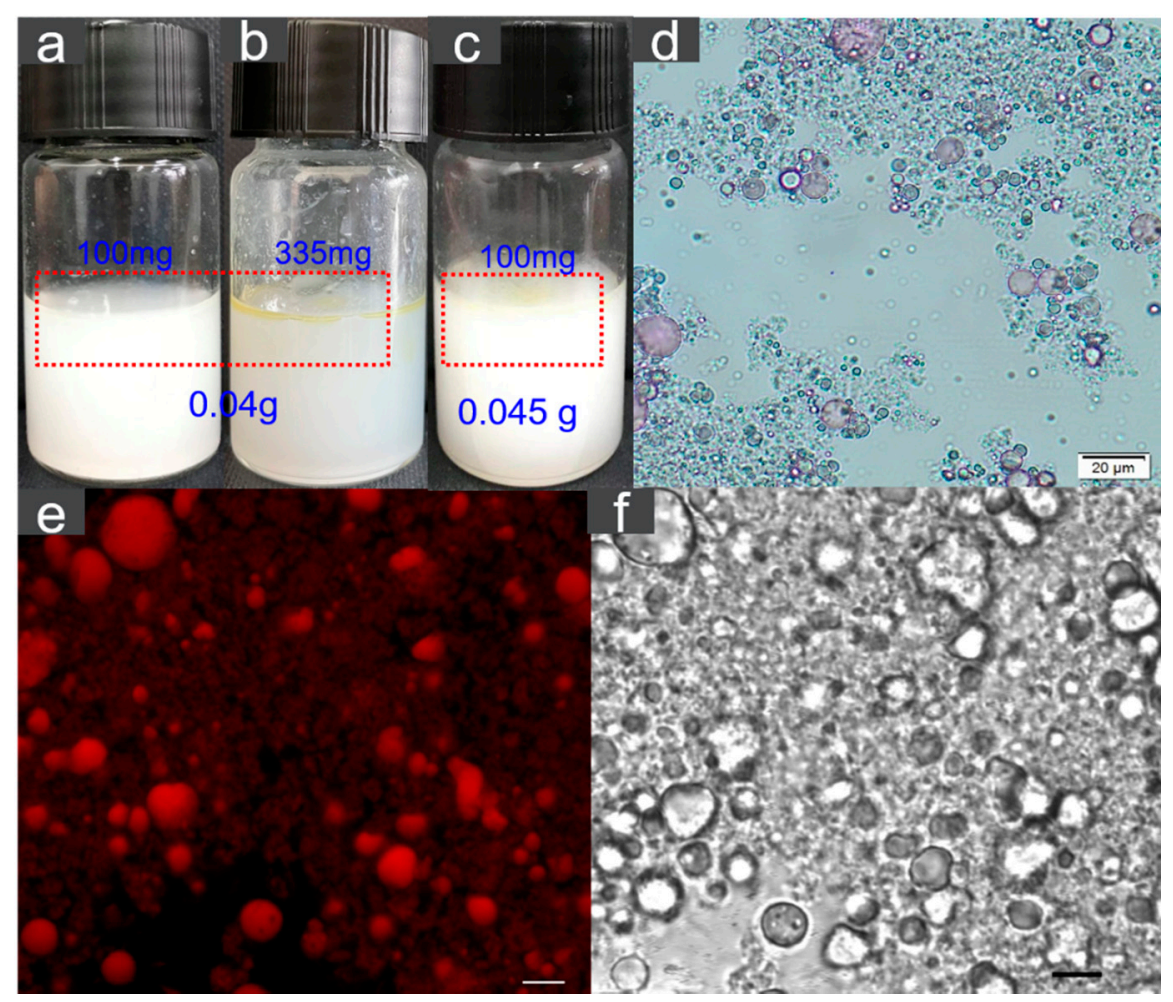

Figure 3. When the addition of SEO was $0.04 \mathrm{~g}$, the oil-carrying performance of organic hollow mesoporous silica formed when the amounts of CTAC were $100 \mathrm{mg}$ (a) and $335 \mathrm{mg}$ (b), respectively. When the addition of SEO was 0.045 , the oil-carrying performance of organic hollow mesoporous silica formed when the amount of CTAC was $100 \mathrm{mg}$ (c). Organic hollow mesoporous silica Pickering emulsion with the highest oil loading was characterised by (d) microscope image, (e) CLSM dark field and (f) CLSM bright field, in which the scales of $\mathrm{c}$ and $\mathrm{d}$ were $10 \mu \mathrm{m}$.

In short, through the above single factor experiment, it was concluded that when the $3.5 \mathrm{~mL}$ of silicon source deposited on the shell, $3 \mathrm{~mL}$ of ammonium hydroxide solution, $30 \%(v / v)$ of OTMS to silicon source deposited on the shell and $100 \mathrm{mg}$ of CTAC were selected, respectively, the prepared organic hollow mesoporous silica had the highest loading capacity $(40 \%(w / w))$.

\subsection{Micro-Morphological Characterisation of Organic Hollow Mesoporous Silica}

Figure $4 \mathrm{a}$ shows the TEM image of hollow mesoporous silica with the highest oilloading. The inner particle diameter was about $175 \mathrm{~nm}$. The water contact angle was $90.4^{\circ}$ (Figure $4 \mathrm{a}$ insert). The high-magnification TEM image (Figure $4 \mathrm{~b}$ ) revealed that the shell of the hollow mesoporous silica displayed an obvious mesoporous structure. The hollow structure was formed by the etching of a sodium carbonate aqueous solution. It was worth noting that the ground particles (Figure $4 \mathrm{c}$ insert) clearly showed a hollow structure, which was quite consistent with the TEM observations. The microspheres in the SEM image (Figure $4 \mathrm{~d}$ ) were measured through the particle size statistics software (Nano Measurer 1.2) to obtain the average particle size of the organic hollow mesoporous silica (Figure $4 \mathrm{~d}$ insert). The average particle size was $222 \mathrm{~nm}$, which was consistent with the results of TEM and SEM. The pore size distribution curve and adsorption isotherm image 
were obtained by Brunner-Emmet-Teller (BET), as shown in Figure 4e. The total volume of pores was $3.354 \mathrm{~cm}^{3} \mathrm{~g}^{-1}$, which was determined with density functional (DFT) methods. According to Barret-Joyner-Halenda (BJH) desorption summary, the surface area and pore volume of mesopores were $86.48 \mathrm{~m}^{2} \mathrm{~g}^{-1}$ and $0.188 \mathrm{~cm}^{3} \mathrm{~g}^{-1}$, respectively. The micropore width, micropore volume and micropore surface area of the hollow mesoporous silica were $1.45 \mathrm{~nm}, 0.118 \mathrm{~cm}^{3} \mathrm{~g}^{-1}$ and $284.6 \mathrm{~m}^{2} \mathrm{~g}^{-1}$, respectively, which were determined with the Langmuir method. According to the classification of IUPAC, the nitrogen adsorption isotherm showed an IV-type curve, and there was an H3-type hysteresis curve in the $\mathrm{P} / \mathrm{P}_{0}$ range, which was steeper than the adsorption branch. The results showed that the shell had a typical hollow structure with nano-mesoporous channel connectivity. The (BJH) pore size distribution curve showed two peaks. The first peak corresponds to the mesoporous structure with an average pore size of $3.3 \mathrm{~nm}$. The pore size distribution of the peak indicated the mesoporous structure. The last peak also indicated a mesopore distribution near $10 \mathrm{~nm}$, which may be attributed to the hollow space of organic hollow silica. This further proved the coexistence of the mesoporous structure and hollow structure of organosilicon [37,38]. The XRD pattern (Figure 4f) showed a diffraction peak around $2 \theta=3^{\circ}$, which was similar to the results of the reported mesoporous silica structure [37,39], further proving the mesostructure in the shells. The result was consistent with the channel distribution curve observed in Figure 4e (inset diagram) and Figure $4 \mathrm{~b}$.

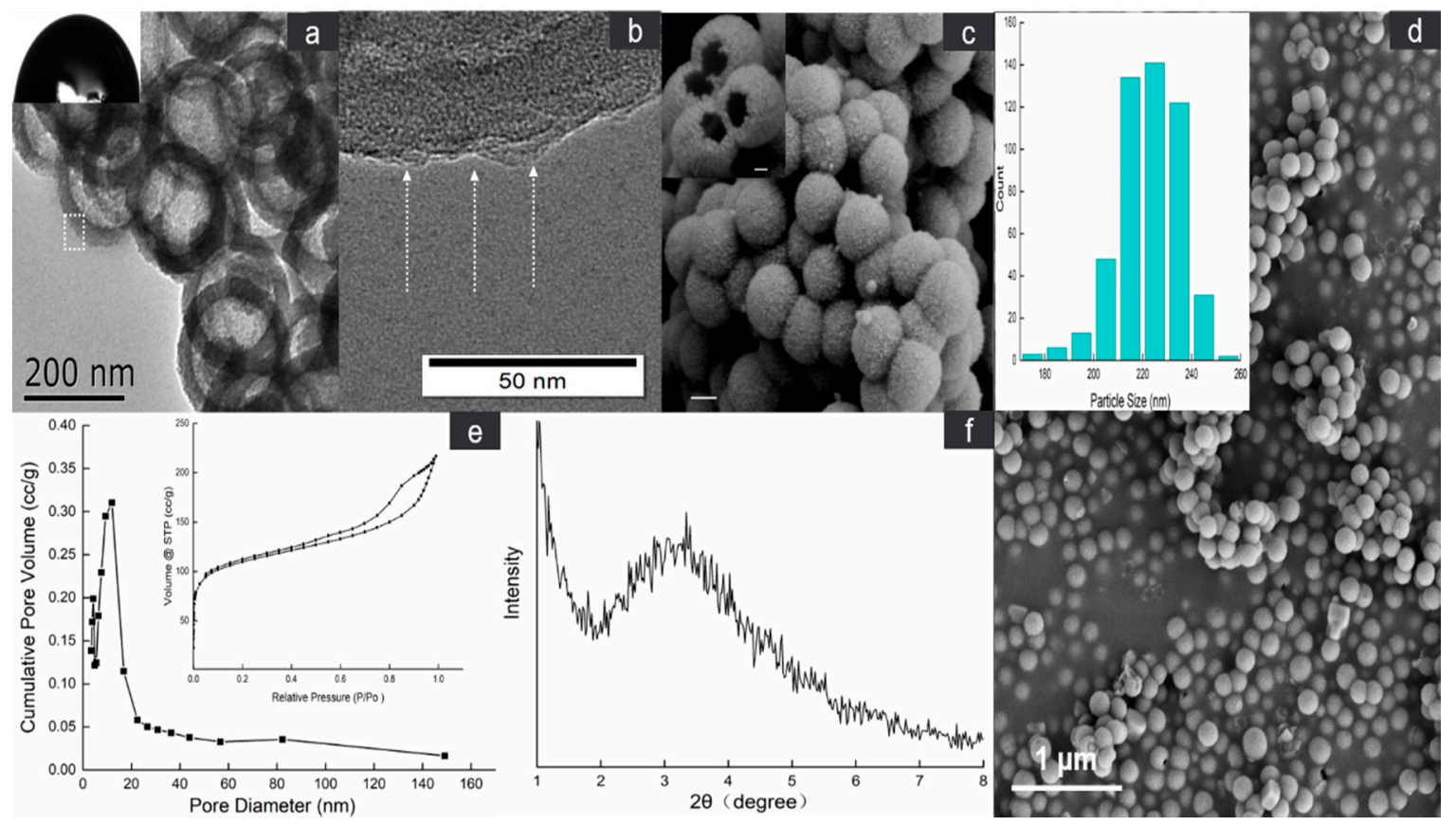

Figure 4. (a,b) TEM,(c,d) SEM, (e) pore size distribution curve under desorption pattern, and (f) SMXRD images of organic hollow mesoporous silica with the highest oil-loading r. Insert in (a) shows WCA image. The scale in (c) was $100 \mathrm{~nm}$. Insert in (c) shows a partially crushed sphere, in which the scale was 50nm. Insert in (d) shows the average particle size. Insert in (e) shows the isotherm image.

To explore the elemental composition on the surface of organic hollow mesoporous silica, it was tested by XPS and EDS. As shown in Figure 5a, a group of peaks for $\mathrm{Si} 2 \mathrm{p}, \mathrm{C}$ s and $\mathrm{O} 1 \mathrm{~s}$ can be observed near $103 \mathrm{eV}, 284 \mathrm{eV}$ and $532 \mathrm{eV}$, respectively. Energy-dispersive $\mathrm{X}$-ray (EDX) analysis (Figure 5b-f) showed that carbon, oxygen and silicon were located on the shell. XPS and EDX elemental distribution images showed that C, O and Si appeared on organic hollow mesoporous silica, indicating that OTMS and TEOS were hydrolysed and condensed successfully to form the silicon shell. 


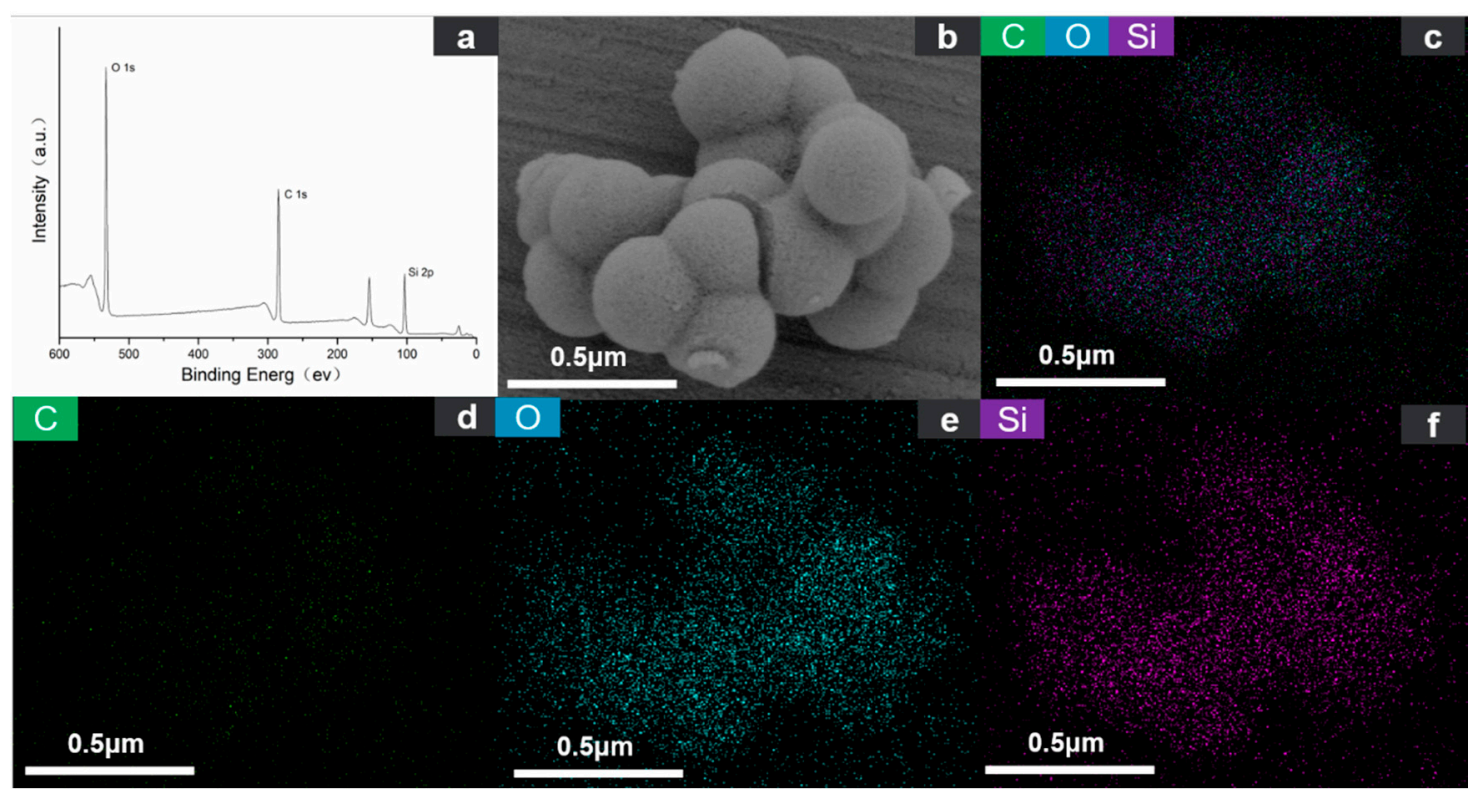

Figure 5. (a) XPS, and (b-f) EDX element distribution images of organic hollow mesoporous silica with the highest oil-loading and dispersion in water. Green, blue and purple denoted C, O and Si, respectively. The scales in (b-f) was $0.5 \mu \mathrm{m}$.

\subsection{FTIR Analysis of Organic Hollow Mesoporous Silica and Its SEO Microcapsules}

The organic hollow mesoporous silica with the highest oil-loading and dispersion in water was prepared. Then, organic hollow mesoporous silica Pickering emulsion was further prepared using this material. The organic hollow mesoporous silica SEO capsules were obtained by the freeze-drying method. The molecular structure and intermolecular force of SEO, organic hollow mesoporous silica and its SEO microcapsule were characterised by FTIR. As shown in Figure 6 and Table 1, the main characteristic peaks were marked in the spectrum.

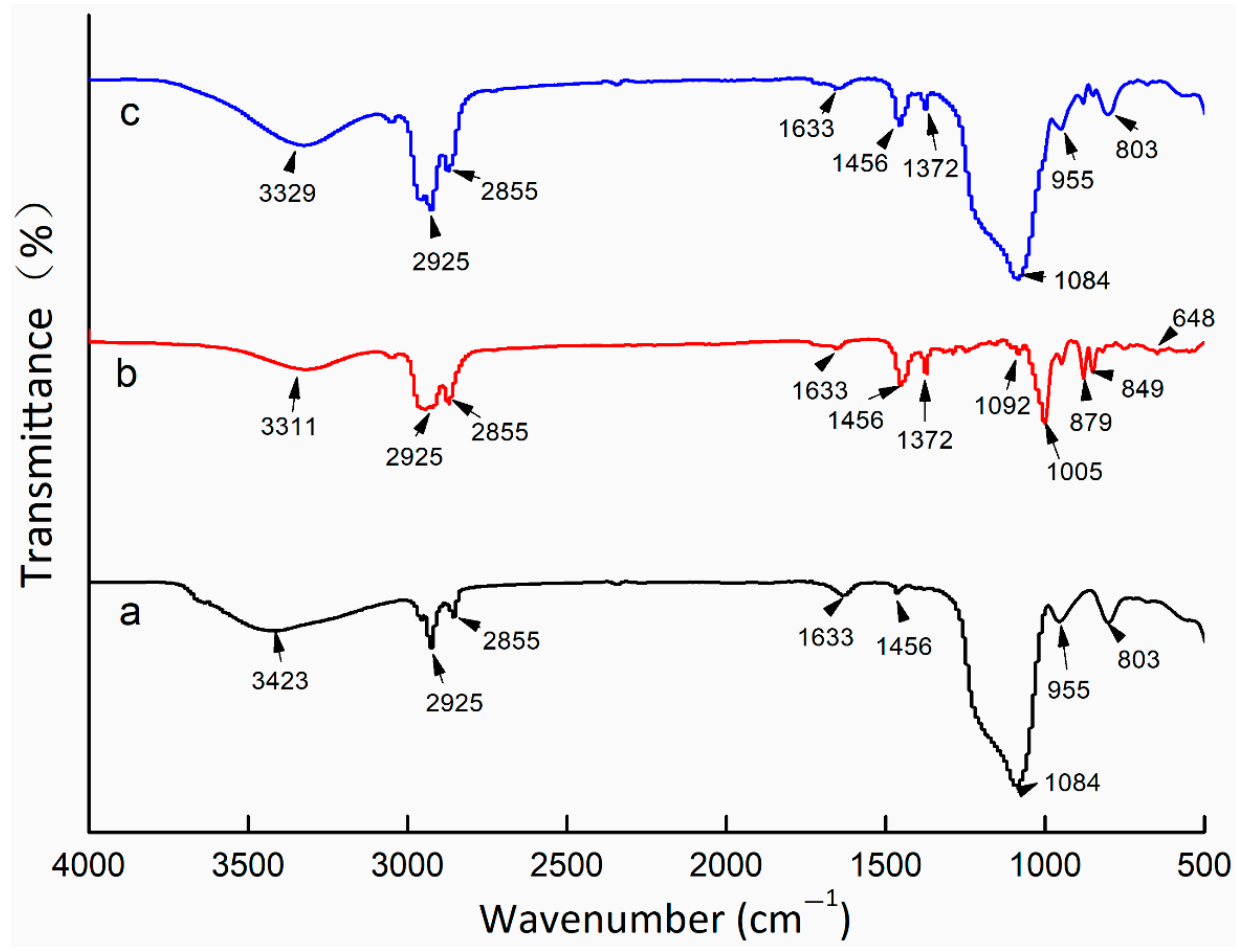

Figure 6. FTIR of organic hollow mesoporous silica blank wall material (a), SEO (b) and organic hollow mesoporous silica SEO microcapsule (c). 
Table 1. Vibrational band shifts and their respective assignments in Fourier transform infrared spectroscopy.

\begin{tabular}{|c|c|c|c|}
\hline \multicolumn{3}{|c|}{ Wavenumber $\left(\mathrm{cm}^{-1}\right)$} & \multirow{2}{*}{ The Reason for the Change of Wavenumber } \\
\hline a & b & c & \\
\hline 3423 & 3311 & 3329 & the stretching vibration of $\mathrm{O}-\mathrm{H}$ \\
\hline 2925 & 2925 & 2925 & \multirow{2}{*}{$\begin{array}{l}\text { the antisymmetric and symmetrical stretching vibration of the } \\
\text { functional group }-\mathrm{CH}_{2-}\end{array}$} \\
\hline 2855 & 2855 & 2855 & \\
\hline 1633 & 1633 & 1633 & the bending vibration of $\mathrm{H}_{2} \mathrm{O}$ \\
\hline \multirow[t]{3}{*}{1456} & 1456 & 1456 & \multirow{2}{*}{$\begin{array}{l}\text { the asymmetric angular vibration and symmetrical angular } \\
\text { vibration of } \mathrm{CH}_{3}\end{array}$} \\
\hline & 1372 & 1372 & \\
\hline & \multicolumn{2}{|l|}{1092} & $\begin{array}{l}\text { the stretching vibrations of the } \mathrm{C}-\mathrm{OH} \text { group in the } \\
\text { alcohol molecule }\end{array}$ \\
\hline \multirow[t]{2}{*}{1084} & & 1084 & the stretching vibrations of -Si-O-Si- group \\
\hline & \multicolumn{2}{|l|}{1005} & $\begin{array}{l}\text { the stretching vibrations of the } \mathrm{C}-\mathrm{OH} \text { group in the } \\
\text { alcohol molecule }\end{array}$ \\
\hline \multirow[t]{2}{*}{955} & & 955 & the stretching vibrations of -Si-O-Si- group \\
\hline & \multicolumn{2}{|l|}{879,849} & the bending vibration absorption of the $\mathrm{C}-\mathrm{H}$ group \\
\hline \multirow[t]{2}{*}{803} & & 803 & the stretching vibrations of -Si-O-group \\
\hline & 648 & & $\begin{array}{l}\text { the stretching vibrations of the } \mathrm{C}-\mathrm{OH} \text { group in the } \\
\text { alcohol molecule }\end{array}$ \\
\hline
\end{tabular}

In spectrum 6 (a), there was a typical broad absorption peak corresponding to the stretching vibration of Si-OH near $3423 \mathrm{~cm}^{-1}$, while the absorption peaks around $2925 \mathrm{~cm}^{-1}$ and $2855 \mathrm{~cm}^{-1}$ were attributed to the antisymmetric and symmetrical stretching vibration of the functional group - $\mathrm{CH}_{2}$ - in OTMS, indicating the existence of organic group - $\mathrm{CH}_{2}$ - in organic hollow mesoporous silica. The absorption peak at $1456 \mathrm{~cm}^{-1}$ was caused by the bending vibration of the $\mathrm{CH}_{3}$ group. The absorption peaks near $1084 \mathrm{~cm}^{-1}$ and $803 \mathrm{~cm}^{-1}$ were attributed to the stretching vibrations of the -Si-O-Si- group and Si-O group [40], while -Si-O-Si- and Si-O were the characteristic functional groups of organic silicon compounds. The appearance of absorption peaks of $2925 \mathrm{~cm}^{-1}, 2855 \mathrm{~cm}^{-1}, 1456 \mathrm{~cm}^{-1}$ and $1084 \mathrm{~cm}^{-1}$, $803 \mathrm{~cm}^{-1}$ indicated that the hydrolysis and condensation reaction between OTMS and TEOS was performed, and organic hollow mesoporous silica was successfully formed.

Compared with the spectra in (a-c) in Figure 6, it was obvious to observe that the broad absorption peak of the O-H group near $3423 \mathrm{~cm}^{-1}$ shifted to low frequency, and the vibration amplitude increased, which was due to the stronger intermolecular or intramolecular hydrogen bonding formed between O-H groups in the compound molecule. Although the three spectra showed obvious absorption peaks near $2925 \mathrm{~cm}^{-1}$ and $2855 \mathrm{~cm}^{-1}$, the strength in spectra $c$ was higher than that in $a$ and $b$, which indicated that SEO was successfully adsorbed in the cavity of organic hollow mesoporous silica, and the vibration amplitude was increased. In the spectra of $\mathrm{a}, \mathrm{b}$ and $\mathrm{c}$, a typical absorption band at $1633 \mathrm{~cm}^{-1}$ was assigned to the bending vibration of $\mathrm{H}_{2} \mathrm{O}$ [27]. The changes at $1456 \mathrm{~cm}^{-1}$ and $1372 \mathrm{~cm}^{-1}$ in Figure $6 \mathrm{~b}, \mathrm{c}$ may be attributed to the asymmetric angular vibration and symmetrical angular vibration of $\mathrm{CH}_{3}$, which might be caused by the destruction of the state of the Pickering emulsion during drying, resulting in the exposure of some SEO to the outside of organic hollow mesoporous silica. In spectrum 6 (b), the stretching vibrations of the $\mathrm{C}-\mathrm{OH}$ group in the alcohol molecule, which were near $1092 \mathrm{~cm}^{-1}, 1005 \mathrm{~cm}^{-1}$ and $648 \mathrm{~cm}^{-1}$, all disappeared in spectrum c. Meanwhile, the bending vibration absorptions of the $\mathrm{C}-\mathrm{H}$ group, which were near $879 \mathrm{~cm}^{-1}$ and $849 \mathrm{~cm}^{-1}$, were weakened. The changes in the characteristic peaks above showed that SEO was successfully adsorbed in the cavity of organic hollow mesoporous silica. 


\subsection{Thermal Stability of SEO, Organic Hollow Mesoporous Silica and Organic Hollow Mesoporous Silica SEO Microcapsule}

The TG curve and DTG curve of SEO $\left(a, a^{\prime}\right)$, organic hollow mesoporous silicon $\left(b, b^{\prime}\right)$ and organic hollow mesoporous silica SEO microcapsule (c,c') had been shown in Figure 7. As can be seen from curve a, the quality of SEO gradually disappeared with the increase of temperature. Most of the mass loss was in the range of $50-186^{\circ} \mathrm{C}$. The maximum thermal decomposition rate occurred at $160^{\circ} \mathrm{C}$, as shown in curve $\mathrm{a}^{\prime}$. The mass loss of curves $\mathrm{b}$ and C also occurred at $65-70{ }^{\circ} \mathrm{C}$, which may be caused by the volatilisation of water molecules in the organic hollow mesoporous silica cavity. The mass loss of curve b was about $22 \%$ at $130-230{ }^{\circ} \mathrm{C}$. It can be seen from Figure $7 \mathrm{~b}^{\prime}$ that the mass rate of this stage decreased quickly. The boiling point of sandalwood alcohol, the main component of SEO, was about $168^{\circ} \mathrm{C}$. The thermal instability of SEO made it released from the cavity of organic hollow mesoporous silica. However, due to the protection of organic hollow mesoporous silica, the volatilisation rate of SEO reached the maximum at $191^{\circ} \mathrm{C}$. Compared with curve a, the thermal stability of SEO wrapped by organic hollow mesoporous silica was obviously improved. The losses of curve $b$ and curve $c$ at $470-545^{\circ} \mathrm{C}$ were $23.8 \%$ and $17 \%$, respectively. The mass loss at this stage may be due to the decomposition of carbon in organic hollow mesoporous silica. This showed that the thermal stability of organic hollow mesoporous silica was excellent. In summary, through the TG and DTG patterns, it can be seen that the initial volatilisation temperature of SEO was $50^{\circ} \mathrm{C}$, and the volatilisation rate reached the maximum at $160{ }^{\circ} \mathrm{C}$, and almost evaporated at $186^{\circ} \mathrm{C}$. On the other hand, the SEO encapsulated by silica began to volatilise at $130{ }^{\circ} \mathrm{C}$, the fastest volatilisation rate was at $191{ }^{\circ} \mathrm{C}$, and then evaporated at $230^{\circ} \mathrm{C}$. Therefore, SEO was encapsulated by organic hollow mesoporous silica, which can not only reduce the volatility of SEO, but also improve the thermal stability of SEO.

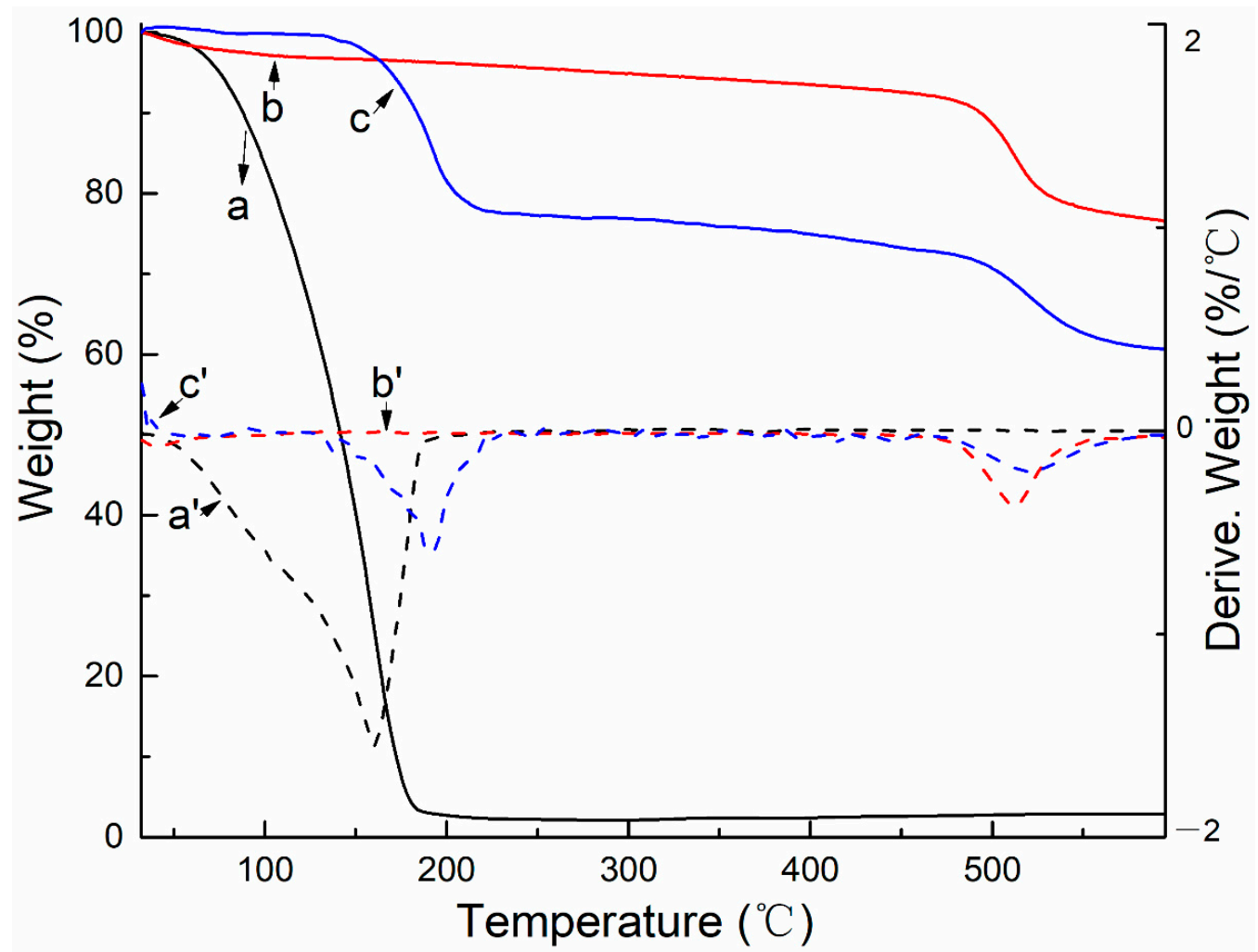

Figure 7. TG and DTG curves of SEO $\left(\mathbf{a}, \mathbf{a}^{\prime}\right)$, organic hollow mesoporous silica $\left(\mathbf{b}, \mathbf{b}^{\prime}\right)$ and organic hollow mesoporous silica SEO microcapsule $\left(\mathbf{c}, \mathbf{c}^{\prime}\right)$, respectively. 


\subsection{Stability of the Pickering Emulsion with Various Organic Hollow Mesoporous Silica Concentrations}

Figure 8 showed the variation of particle size and PDI of Pickering emulsion of organic mesoporous silica with different concentrations over time. With the increase of the concentration of organic hollow mesoporous silica, the stability of Pickering emulsion increased from $0 \mathrm{~h}$ (Figure 8a) to $21 \mathrm{~h}$ (Figure 8c), while the particle size decreased from $8.95 \mu \mathrm{m}$ to $8.78 \mu \mathrm{m}$. This may be due to the increase of particle concentration in the system - enough particles were adsorbed at the interface and form a dense interface film, which improved the surface coverage and led to the formation of network structure around the emulsion droplets. This structure formed the skeleton of the emulsion and inhibited the coalescence of droplets, further improving the stability of the emulsion [41,42]. From Figure $8 \mathrm{c}$, it was observed that the particle size increased with time. PDI was relatively stable within $21 \mathrm{~h}$, but increased suddenly at $24 \mathrm{~h}$. The photos (Figure 8c) of the emulsion also showed that delamination began to occur at $24 \mathrm{~h}$. These results showed that the organic hollow mesoporous silica Pickering emulsion can be stabilised for $21 \mathrm{~h}$ at room temperature.

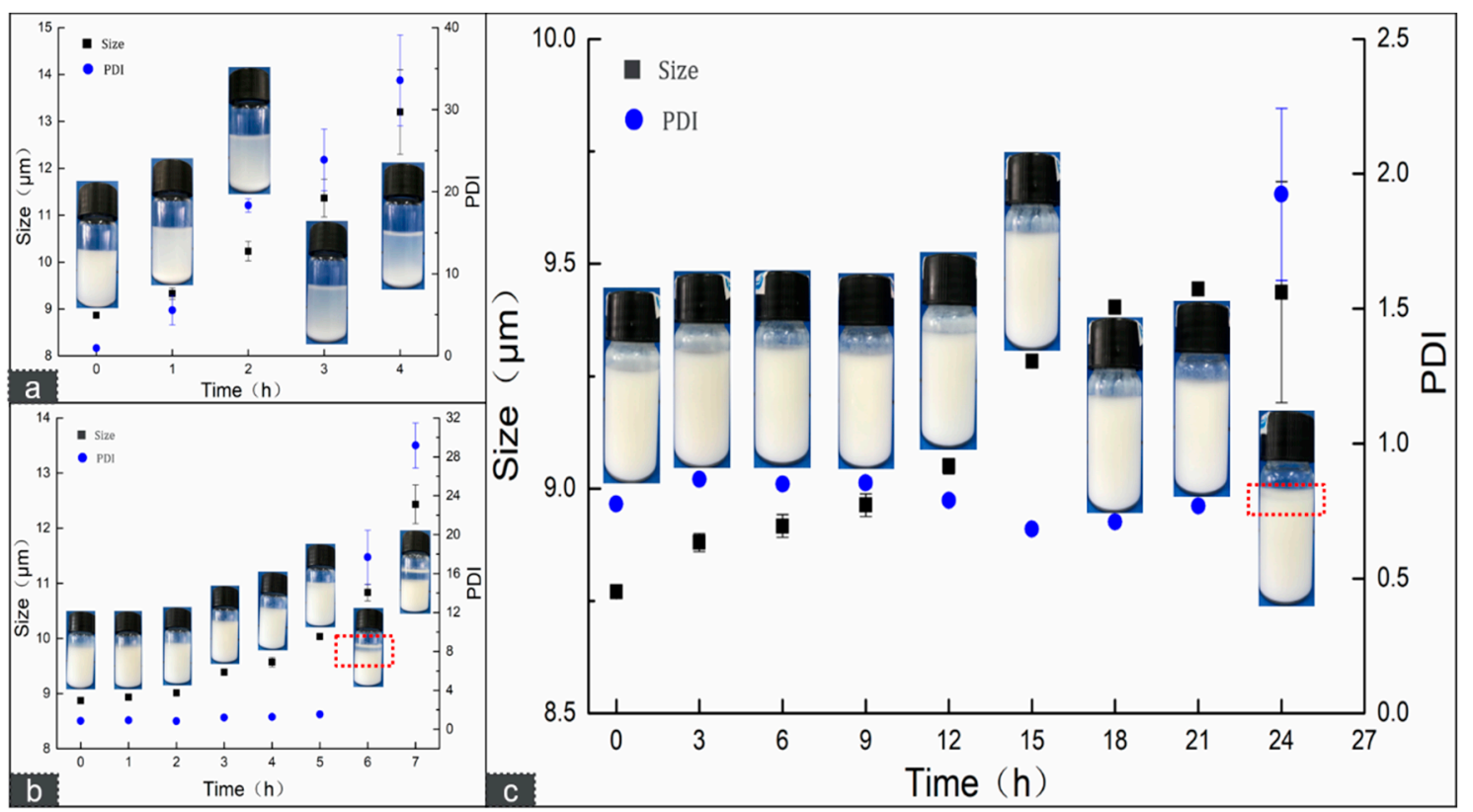

Figure 8. Variation of particle size and PDI of Pickering emulsion of organic mesoporous silica with different concentrations over time. The concentrations in Figure (a-c) were 1\%, 10\% and 20\%, respectively.

\section{Materials and Methods}

\subsection{Chemicals and Materials}

Cetyltrimethylammonium chloride (99\%, CTAC), tetraethoxysilane $(99 \%$, TEOS) and n-octyltrimethoxysilane $(97 \%+$, OTMS) were kindly provided by Adamas-beta, Shanghai Titan Scientific Co., Ltd. Ammonium hydroxide solution (AR, 25-28\%) and ethanol anhydrous ( $\geq 99.7 \%$, AR) were purchased from General-reagent, Shanghai Titan Scientific Co., Ltd (Shanghai, China). Sodium carbonate anhydrous (AR) and hydrochloric acid (AR, $\mathrm{HCl}$ ) were from Sinopharm Chemical Reagent Co., Ltd (Shanghai, China). Nile Red (99\%, pure) and SEO were obtained from Shanghai Titan Scientific Co., Ltd. Deionized water made by Shanghai Institute of Technology was used in all experiments.

\subsection{Preparation of Organic Hollow Mesoporous Silica}

According to the improved Stöber method [43], $10 \mathrm{~mL}$ deionised water, $70 \mathrm{~mL}$ ethanol anhydrous and a certain amount of ammonium hydroxide solution were successively poured into a conical bottle with a magnetic agitator, and the mixture was stirred at $600 \mathrm{rpm}$ for about $30 \mathrm{~min}$ at $35^{\circ} \mathrm{C}$. The initial $\mathrm{pH}$ of the reaction was 11 . After this, $6 \mathrm{~mL}$ 
of TEOS was added to the mixture. Subsequently, the reaction solution was stirred for $1 \mathrm{~h}$ to form a milky silica colloidal suspension. Following, a certain amount of CTAC was added. After stirring for $30 \mathrm{~min}$, a certain proportion of the mixture of OTMS and TEOS used as silicon source was added. The hydrolysis and condensation of TEOS and OTMS reacted for $1.5 \mathrm{~h}$. At last, through centrifugation and washing (three times), the white powders of organic bilayer silica microspheres were successfully prepared. Then $3.75 \mathrm{~g}$ of the obtained product was dispersed in $100 \mathrm{~mL} 21.2 \mathrm{mg} / \mathrm{mL}$ sodium carbonate aqueous solution and stirred at $80^{\circ} \mathrm{C}$ for $50 \mathrm{~min}$ to convert the products into organic hollow silica microspheres. After centrifugation and washing, they were mixed into $200 \mathrm{~mL}$ ethanol solution containing $1.6 \mathrm{~mL}$ concentrated hydrochloric acid and refluxed at $125^{\circ} \mathrm{C}$ for $3 \mathrm{~h}$ to remove CTAC. Eventually, through centrifugation, washing and vacuum drying, the final product of organic hollow mesoporous silica was obtained.

\subsection{Preparation of a Solution System of Organic Hollow Mesoporous Silica and SEO}

$0.06 \mathrm{~g}$ organic hollow mesoporous silica powder was ultrasonically dispersed in $9.9 \mathrm{~mL}$ deionised water, and SEO was added. The emulsions of organic hollow mesoporous silica and SEO were obtained by magnetic stirring with $500 \mathrm{rpm} / \mathrm{min}$ at $25^{\circ} \mathrm{C}$ and mixed by vortexing for $3 \mathrm{~h}$.

\subsection{Stability of the Pickering Emulsion with Various Organic Hollow Mesoporous Silica Concentrations}

Although the loading capacity and dispersion of the emulsion obtained from the single factor test are the best, its stability is not so satisfactory. It is reported that the stability of the emulsion is closely related to the wettability, size, shape, surface properties and particle concentration of the particles [42]. In order to get a more stable emulsion, the concentration of the particles was changed. $0.06 \mathrm{~g}$ hollow mesoporous silica powder was uniformly dispersed in $9.9 \mathrm{~mL}$ deionised water. Then $0.04 \mathrm{~g}$ SEO was added. The Organic hollow mesoporous silica with a concentration of $1 \%$ was obtained by magnetic stirring with $500 \mathrm{rpm} / \mathrm{min}$ at $25^{\circ} \mathrm{C}$ and mixed by vortexing for $3 \mathrm{~h}$. Using the same method, organic hollow mesoporous silica Pickering emulsions with concentrations of $10 \%$ and $20 \%$ were prepared, respectively.

\subsection{Characterisation of Micro-Morphology and Physicochemical Properties of Organic Hollow Mesoporous Silica}

Transmission Electron Microscopy (TEM) images were acquired using a jeol2100f TEM with a field emission gun operated at $200 \mathrm{kV}$. Microparticle morphology was observed by field-emission SEM (Sigma 300, Oberkochen, Germany). The dried powder samples were fixed on a metal stub with the aid of double-sided tape, then sputter-coated with gold. Finally, it was detected by Inlens and ET secondary electron detectors and Smart EDX spectrometer, and the images were obtained on a field-emission SEM at $15.0 \mathrm{kV}$. $\mathrm{N}_{2}$ physical adsorption was performed on accelerated surface area and porosity analyser (ASAP 2460) under a continuous adsorption condition. Pore diameters were calculated from the desorption branch of the isotherm with the BJH method. Small-angle X-ray diffraction (SAXRD) patterns of the prepared materials were noted down with a Japanese Neo-Rigaku Ultimate IV diffractometer. The model K-Alpha (Thermo Fisher) X-ray photoelectron spectrometer (XPS) was used to detect the organic hollow mesoporous silica and determine the elemental composition of its surface. The water contact angle (WCA) was measured by Theta flex optical contact angle meter (Biolin scientific AB, Gothenburg, Sweden). The droplet volume is $5 \mu \mathrm{L}$, and the angle accuracy is $\pm 0.5^{\circ}$. Before the test, the organic hollow mesoporous silica was pressed into a uniform sheet by a pressing machine (FW-4, Tianjin skylight new optical instrument technology CO., LTD, Tianjin, China) under the pressure of $20 \mathrm{MPa}$, and then tested. 


\subsection{Characterisation of Morphology and Properties of Organic Hollow Mesoporous Silica Pickering Emulsion}

$0.04 \mathrm{~g}$ of SEO marked with Nile red was mixed with $9.9 \mathrm{~mL}$ water and $0.06 \mathrm{~g}$ uniform solution of organic hollow mesoporous silica. After mixing by vortexing for $3 \mathrm{~h}$, the morphology of the emulsion was observed by Olympus microscope (model: BX53, Shanghai, China) and confocal laser scanning microscope (CLSM, model: FV1200, Shanghai, China). The Fourier transform infrared (FTIR) analysis was carried out on Thermo Scientific Nicolet iS5 infrared spectrometer. Malvern laser particle size analyser Mastersizer 3000 and static observation method were used to characterise the stability of the Organic Hollow mesoporous silica Pickering Emulsion. Thermal stability results were tested by a thermogravimetric (TG) analyser (Netzsch STA 449F3, Selb, Germany).

\section{Conclusions}

In this study, organic hollow mesoporous silica was prepared using OTMS as an organic hydrophobic agent. The effects of the amount of silicon source deposited on the shell, the addition of ammonium hydroxide solution, the proportion of OTMS and the addition of CTAC on the WCA of organic hollow mesoporous silica and its loading capacity to SEO were investigated. We found that organic hollow mesoporous silica Pickering emulsion can be relatively stable at room temperature for $21 \mathrm{~h}$ without using any surfactant, and the loading capacity can be up to $40 \%(w / w)$. Sandalwood essential oil (SEO), which is hydrophobic, was successfully dispersed in the aqueous phase, overcoming the shortcomings of poor water solubility. At the same time, the TG images showed that the organic hollow mesoporous silica improved the volatility and thermal stability of SEO. SEO can not only be encapsulated in the cavity of organic hollow mesoporous silica microspheres, but also can form a Pickering emulsion with organic hollow mesoporous silica microspheres, which further increases the loading capacity of SEO. This system can act as a microplastic substitute with broad prospects in the cosmetics and personal care industry, laying a foundation for the preparation and applications of high loading capacity microcapsules in aqueous media. In addition, we will continue to improve the system to prolong its stability so that the aroma in daily chemical products can be released in multiple stages and remain fragrant for an extended time.

Author Contributions: Data curation, methodology, writing—original draft and writing-review and editing, H.B.; Funding acquisition, Z.X. and Y.N.; Project administration, X.K.; Software, H.B. and Y.B.; Validation, S.J. All authors have read and agreed to the published version of the manuscript.

Funding: This research was supported by National Key Research and Development Program Nanotechnology Specific Project (2016YFA0200304), National Natural Science Foundation of China (21776178, 31901618), Open project fund from Shanghai Collaborative Innovation Center for Aroma, Perfume and Cosmetics Research, and Science and Technology Commission Shanghai Municipality Technical Standard Program (18DZ2200400).

Institutional Review Board Statement: Not applicable.

Informed Consent Statement: Not applicable.

Data Availability Statement: Not applicable to this article.

Conflicts of Interest: There is no conflict of interest to declare.

Sample Availability: Samples of the compounds are available from the authors.

\section{References}

1. Cui, H.; Wu, J.; Li, C.; Lin, L. Promoting anti-listeria activity of lemongrass oil on pork loin by cold nitrogen plasma assist. J. Food Saf. 2016, 37, e12316. [CrossRef]

2. Yao, W.; Wang, S.; Chen, Y.; Wang, H. Composition and Antibacterial Activity of Essential Oils of Flos Sophorae immaturus. Int. J. Food Prop. 2011, 14, 903-913. [CrossRef]

3. Burdock, G.A.; Carabin, I.G. Safety assessment of sandalwood oil (Santalum album L.). Food Chem. Toxicol. 2008, 46, 421-432. [CrossRef] 
4. Herculano, E.D.; de Paula, H.C.; de Figueiredo, E.A.; Dias, F.G.; Pereira, V.D.A. Physicochemical and antimicrobial properties of nanoencapsulated Eucalyptus staigeriana essential oil. LWT 2015, 61, 484-491. [CrossRef]

5. Wang, Y.-F.; Jia, J.-X.; Tian, Y.-Q.; Shu, X.; Ren, X.-J.; Guan, Y.; Yan, Z.-Y. Antifungal effects of clove oil microcapsule on meat products. LWT 2018, 89, 604-609. [CrossRef]

6. Júnior, O.T.; Kuhn, F.; Padilha, P.J.M.; Vicente, L.R.M.; Costa, S.W.; Boligon, A.A.; Scapinello, J.; Nesi, C.N.; Magro, J.D.; Castellví, S.L. Microencapsulation of essential thyme oil by spray drying and its antimicrobial evaluation against Vibrio alginolyticus and Vibrio parahaemolyticus. Braz. J. Biol. 2017, 78, 311-317. [CrossRef]

7. He, L.; Hu, J.; Deng, W. Preparation and application of flavor and fragrance capsules. Polym. Chem. 2018, 9, 4926-4946. [CrossRef]

8. Fendall, L.S.; Sewell, M.A. Contributing to marine pollution by washing your face: Microplastics in facial cleansers. Mar. Pollut. Bull. 2009, 58, 1225-1228. [CrossRef] [PubMed]

9. Hu, D.; Shen, M.; Zhang, Y.; Zeng, G. Micro(nano)plastics: An unignorable carbon source? Sci. Total. Environ. 2019, 657, 108-110. [CrossRef] [PubMed]

10. Li, W.; Cai, Y.; Zhong, Q.; Yang, Y.; Kundu, S.C.; Yao, J. Silk sericin microcapsules with hydroxyapatite shells: Protection and modification of organic microcapsules by biomimetic mineralization. J. Mater. Chem. B 2016, 4, 340-347. [CrossRef]

11. Kresge, C.T.; Leonowicz, M.E.; Roth, W.J.; Vartuli, J.C.; Beck, J.S. Ordered mesoporous molecular sieves synthesized by a liquid-crystal template mechanism. Nat. Cell Biol. 1992, 359, 710-712. [CrossRef]

12. Berry, J.D.; Mettu, S.; Dagastine, R.R. Precise measurements of capsule mechanical properties using indentation. Soft Mater. 2017, 13, 1943-1947. [CrossRef] [PubMed]

13. Veith, S.R.; Hughes, E.; Pratsinis, S.E. Restricted diffusion and release of aroma molecules from sol-gel-made porous silica particles. J. Control. Release 2004, 99, 315-327. [CrossRef]

14. Lin, Y.; Zhao, A.; Tao, Y.; Ren, J.; Qu, X. Ionic Liquid as an Efficient Modulator on Artificial Enzyme System: Toward the Realization of High-Temperature Catalytic Reactions. J. Am. Chem. Soc. 2013, 135, 4207-4210. [CrossRef] [PubMed]

15. Guidotti, M.; Pirovano, C.; Ravasio, N.; Lázaro, B.; Fraile, J.M.; Mayoral, J.A.; Coq, B.; Galarneau, A. The use of $\mathrm{H}_{2} \mathrm{O}_{2}$ over titanium-grafted mesoporous silica catalysts: A step further towards sustainable epoxidation. Green Chem. 2009, 11, 1421-1427. [CrossRef]

16. Lu, Z.; Wang, J.; Qub, L.; Kanb, G.; Zhangac, T.; Shenac, J.; Lia, Y.; Yanga, J.; Niude, Y.; Xiaode, Z.; et al. Reactive mesoporous silica nanoparticles loaded with limonene for improving physical and mental health of mice at simulated microgravity condition. Bioact. Mater. 2020, 5, 1127-1137. [CrossRef]

17. Qu, F.; Zhu, G.; Lin, H.; Sun, J.; Zhang, D.; Li, S.; Qiu, S. Drug Self-Templated Synthesis of Ibuprofen/Mesoporous Silica for Sustained Release. Eur. J. Inorg. Chem. 2006, 2006, 3943-3947. [CrossRef]

18. Wibowo, D.; Zhao, C.-X.; Peters, B.C.; Middelberg, A.P.J. Sustained Release of Fipronil Insecticide in Vitro and in Vivo from Biocompatible Silica Nanocapsules. J. Agric. Food Chem. 2014, 62, 12504-12511. [CrossRef]

19. Dou, J.; Zeng, H.C. Integrated Networks of Mesoporous Silica Nanowires and Their Bifunctional Catalysis-Sorption Application for Oxidative Desulfurization. ACS Catal. 2013, 4, 566-576. [CrossRef]

20. Li, Y.; Li, N.; Pan, W.; Yu, Z.; Yang, L.; Tang, B. Hollow Mesoporous Silica Nanoparticles with Tunable Structures for Controlled Drug Delivery. ACS Appl. Mater. Interfaces 2017, 9, 2123-2129. [CrossRef]

21. Zhu, Y.-F.; Shi, J.-L.; Li, Y.-S.; Chen, H.-R.; Shen, W.-H.; Dong, X.-P. Storage and release of ibuprofen drug molecules in hollow mesoporous silica spheres with modified pore surface. Microporous Mesoporous Mater. 2005, 85, 75-81. [CrossRef]

22. Zhu, Y.; Shi, J.; Shen, W.; Dong, X.; Feng, J.; Ruan, M.; Li, Y. Stimuli-Responsive Controlled Drug Release from a Hollow Mesoporous Silica Sphere/Polyelectrolyte Multilayer Core-Shell Structure. Angew. Chem. Int. Ed. 2005, 44, 5083-5087. [CrossRef] [PubMed]

23. Wang, J.-X.; Wang, Z.-H.; Chen, J.-F.; Yun, J. Direct encapsulation of water-soluble drug into silica microcapsules for sustained release applications. Mater. Res. Bull. 2008, 43, 3374-3381. [CrossRef]

24. Ciriminna, R.; Pagliaro, M. Sol-gel microencapsulation of odorants and flavors: Opening the route to sustainable fragrances and aromas. Chem. Soc. Rev. 2013, 42, 9243-9250. [CrossRef]

25. Dashairya, L.; Barik, D.D.; Saha, P. Methyltrichlorosilane functionalized silica nanoparticles-treated superhydrophobic cotton for oil-water separation. J. Coat. Technol. Res. 2019, 16, 1021-1032. [CrossRef]

26. Cai, Y.; Li, J.; Yi, L.; Yan, X.; Li, J. Fabricating superhydrophobic and oleophobic surface with silica nanoparticles modified by silanes and environment-friendly fluorinated chemicals. Appl. Surf. Sci. 2018, 450, 102-111. [CrossRef]

27. Guo, F.; Wen, Q.; Peng, Y.; Guo, Z. Multifunctional hollow superhydrophobic $\mathrm{SiO}_{2}$ microspheres with robust and self-cleaning and separation of oil/water emulsions properties. J. Colloid Interface Sci. 2017, 494, 54-63. [CrossRef]

28. Hwang, H.S.; Lee, S.B.; Park, I. Fabrication of raspberry-like superhydrophobic hollow silica particles. Mater. Lett. 2010, 64, 2159-2162. [CrossRef]

29. Ye, H.; Zhu, L.; Li, W.; Liu, H.; Chen, H. Constructing Fluorine-Free and Cost-Effective Superhydrophobic Surface with Normal-Alcohol-Modified Hydrophobic SiO2 Nanoparticles. ACS Appl. Mater. Interfaces 2017, 9, 858-867. [CrossRef]

30. Shi, S.; Wang, M.; Chen, C.; Lu, F.; Zheng, X.; Gao, J.; Xu, J. Preparation of hydrophobic hollow silica nanospheres with porous shells and their application in pollutant removal. RSC Adv. 2013, 3, 1158-1164. [CrossRef]

31. Li, S.; Jiao, X.; Yang, H. Hydrophobic Core/Hydrophilic Shell Structured Mesoporous Silica Nanospheres: Enhanced Adsorption of Organic Compounds from Water. Langmuir 2013, 29, 1228-1237. [CrossRef] [PubMed] 
32. Zhang, L.; Wang, L.; Yao, H.; Xu, F.; Chen, Y. Biodegradable and biocompatible monodispersed hollow mesoporous organosilica with large pores for delivering biomacromolecules. J. Mater. Chem. B 2017, 5, 8013-8025. [CrossRef]

33. Chen, Y.; Chen, H.; Guo, L.; He, Q.; Chen, F.; Zhou, J.; Feng, J.; Shi, J. Hollow/Rattle-Type Mesoporous Nanostructures by a Structural Difference-Based Selective Etching Strategy. ACS Nano 2010, 4, 529-539. [CrossRef] [PubMed]

34. Zhang, K.; Chen, H.; Zheng, Y.; Chen, Y.; Ma, M.; Wang, X.; Wang, L.; Zeng, D.; Shi, J. A facile in situ hydrophobic layer protected selective etching strategy for the synchronous synthesis/modification of hollow or rattle-type silica nanoconstructs. J. Mater. Chem. 2012, 22, 12553-12561. [CrossRef]

35. Fang, X.; Chen, C.; Liu, Z.; Liu, P.; Zheng, N. A cationic surfactant assisted selective etching strategy to hollow mesoporous silica spheres. Nanoscale 2011, 3, 1632-1639. [CrossRef] [PubMed]

36. Aouada, F.A.; de Moura, M.R.; Orts, W.J.; Mattoso, L.H.C. Polyacrylamide and methylcellulose hydrogel as delivery vehicle for the controlled release of paraquat pesticide. J. Mater. Sci. 2010, 45, 4977-4985. [CrossRef]

37. Ma, N.; Deng, Y.; Liu, W.; Li, S.; Xu, J.; Qu, Y.; Gan, K.; Sun, X.; Yang, J. A one-step synthesis of hollow periodic mesoporous organosilica spheres with radially oriented mesochannels. Chem. Commun. 2016, 52, 3544-3547. [CrossRef]

38. Teng, Z.; Zhang, J.; Li, W.; Zheng, Y.; Su, X.; Tang, Y.; Dang, M.; Tian, Y.; Yuwen, L.; Weng, L.; et al. Facile Synthesis of YolkShell-Structured Triple-Hybridized Periodic Mesoporous Organosilica Nanoparticles for Biomedicine. Small 2016, 12, 3550-3558. [CrossRef]

39. Okamoto, M.; Tsukada, H.; Fukasawa, S.; Sakajiri, A. Synthesis of hollow and rattle-type mesoporous silica spheres by treating layered mesoporous silica with a basic solution, and using the spheres as microreactors for two-phase reactions. J. Mater. Chem. A 2015, 3, 11880-11890. [CrossRef]

40. Si, Z.; Hu, S.; Cai, D.; Qin, P.; Xu, Q. Performance enhancement of a polydimethylsiloxane membrane for effectiven-butanol pervaporation by bonding multi-silyl-functional MCM-41. RSC Adv. 2018, 8, 5127-5135. [CrossRef]

41. Dugyala, V.R.; Daware, S.V.; Basavaraj, M.G. Shape anisotropic colloids: Synthesis, packing behavior, evaporation driven assembly, and their application in emulsion stabilization. Soft Mater. 2013, 9, 6711-6725. [CrossRef]

42. Wu, J.; Ma, G.-H. Recent Studies of Pickering Emulsions: Particles Make the Difference. Small 2016, 12, 4633-4648. [CrossRef] [PubMed]

43. Stöber, W.; Fink, A.; Bohn, E. Controlled growth of monodisperse silica spheres in the micron size range. J. Colloid Interface Sci. 1968, 26, 62-69. [CrossRef] 